doi:10.17659/01.2019.0035

Journal of Case Reports 2019;9(2):135-138

\title{
Neurosyphilis Presenting with Stroke and Complex Partial Seizure: Temporo- Parieto-Occipital Involvement
}

\author{
Ugur Kostakoglu ${ }^{1}$, Ayse Erturk ${ }^{1}$, Sevda Ozdemir Al ${ }^{1}$, Maksude Esra Kadioglu${ }^{1}$, Mehmet Fatih Inecikli², \\ Ilknur Esen Yildiz ${ }^{1}$ \\ ${ }^{1}$ Department of Infectious Disease and Clinical Microbiology and ${ }^{2}$ Department of Radiology, Recep Tayyip Erdogan University, \\ 53100, Rize, Turkey.
}

\section{Corresponding Author: \\ Dr. Ayse Erturk \\ Email: ayseace25@hotmail.com}

This is an Open Access article distributed under the terms of the Creative Commons Attribution License (creativecommons.org/ licenses/by/3.0).

Received

Accepted

Published

February 11, 2019

June 13, 2019

June 30, 2019

\begin{abstract}
Background: Syphilis is an infectious disease caused by Treponema pallidum with multiple organ involvement in which the central nervous system (CNS) is also affected. Neurosyphilis should be considered in the differential diagnosis of many CNS infections due to its numerous various clinical manifestations. Hemiparesia and complex partial seizures are rare presentations of syphilis. Case Report: A 56 year old male patient was admitted to the emergency department due to fever, syncope and vomiting. His general condition was poor, he was somnolent and partially cooperative, and was disoriented in person and place. MRI resembled HSV encephalitis, mesial temporal syndromes; and increased mononuclear cells in cerebrospinal fluid (CSF) was suggestive for HSV encephalitis. However, CSF and blood tests supported the diagnosis of neurosyphilis. Conclusion: Neurosyphilis should not be overlooked at differential diagnosis in patients presenting with clinical and radiological findings suggestive of herpes encephalitis.
\end{abstract}

Keywords: Encephalitis, Neurosyphilis, Paresis, Seizures, Syphilis.

\section{Introduction}

Syphilis progresses with multiorgan involvement 'closely mimicking' several systemic diseases [1-3]. The disease has primary, secondary, latent, and late (tertiary syphilis) stages. Neurosyphilis is a severe complication that may develop at any time during the course of syphilis [2]. The clinical findings of neurosyphilis are rich and vary depending on whether the area of involvement is parenchymal, vascular or meningeal [4]. At imaging performed to assess clinical manifestations for diagnosis, cerebral gummas, dural-based lesions capable of mimicking meningiomas, and mesial temporal lobe anomalies capable of mimicking herpes encephalitis must be regarded as indicative $[2,4]$.

\section{Case Report}

A 56-year-old male patient was brought to the emergency department due to fever, syncope and vomiting commencing four days previously. He was described as occasionally speaking unintelligibly and exhibiting agitation, followed by normal behaviors. At physical examination his general condition was poor, he was somnolent and partially cooperative, and was disoriented in person and place. No pathology was determined at respiratory, cardiovascular or abdominal examinations. Bilateral, direct and indirect light reflexes were elicited at neurological examination, Babinski's sign was bilaterally negative, and no focal neurological findings were observed. No additional disease was present in his own or family medical histories, although he drank alcohol and smoked (30 packs/year).

Lumbar puncture (LP) was performed. Cerebrospinal fluid (CSF) opening pressure was normal, and CSF was clear in appearance. CSF cell count was 940 erythrocytes and 50 leukocytes 
(60\% polymorphonuclear lymphocytes), and 4-5 leukocytes were observed in every field at Gramstaining. At CSF biochemistry, a glucose level of $53 \mathrm{mg} / \mathrm{dL}$ and simultaneous blood glucose of 107 $\mathrm{mg} / \mathrm{dL}$ were determined. Protein was $71 \mathrm{mg} / \mathrm{dL}$, $\mathrm{Na} 135 \mathrm{mmol} / \mathrm{L}$, and chloride $116 \mathrm{mmol} / \mathrm{L}$, and the patient was hospitalized with a diagnosis of meningoencephalitis. Agitation, hallucination and chewing-type focal seizures developed on the $2^{\text {nd }}$ hour of admission, and with-contrast and diffusion cranial magnetic resonance imaging (MRI) and electroencephalogram (EEG) were performed. Focal epileptic discharges were observed at EEG, while cranial MRI revealed a signal increase in FLAIR series and leptomeningeal mild contrast enhancement in the right temporal parahippocampal area, the insular and subcallosal cortex, and at the level of the superior temporal gyrus cortex. Findings compatible with encephalitis, in the form of occasional suspicious diffusion restriction, were observed on diffusion-weighted images [Fig.1]. Empirical treatment consisting of intravenous (iv) acyclovir $3 \times 750 \mathrm{mg}(5-12.5 \mathrm{mg} / \mathrm{kg})$, ampicillin $6 \times 2 \mathrm{~g}$ iv, and mannitol $4 \times 100 \mathrm{cc}$ iv was initiated. The Brucella agglutination test, Brucella IgM-IgG panel, Borrelia burgdorferi IgM, IgG, Gruber-Widal test, and Venereal Disease Research Laboratory (VDRL) test were also requested for differential diagnosis. CSF culture and acid resistant bacilli (ARB) direct examination, CSF tuberculosis "Polymerase Chain Reaction" (PCR), and the meningitis pathogens PCR panel were requested. VDRL in serum at a $1 / 32$ titer was reported positive, and the T. pallidum hemagglutination (TPHA) test was positive. LP was repeated after $48 \mathrm{~h}$ of monitoring: the CSF cell count was 20 erythrocytes, and 20 leukocytes (60\% PMNL), and 1-2 leukocytes were determined in each field at Gram-staining. At CSF biochemistry, a glucose level of $52 \mathrm{mg} / \mathrm{dL}$ was determined, with a simultaneous blood glucose level of $110 \mathrm{mg} / \mathrm{dL}$. Other values were protein $120 \mathrm{mg} / \mathrm{dL}$, sodium 140 $\mathrm{mmoI} / \mathrm{L}$, and chloride $127 \mathrm{mmoI} / \mathrm{L} . \mathrm{CSF}$ VDRL at a 1/8 titer was reported positive, and CSF TPHA was positive at $1 / 8$ titer. No growth occurred in the first CSF aerobic and anaerobic cultures taken, and $\mathrm{ARB}$ direct examination was negative. Ampicillin was stopped, and the patient was started on 4 million units iv crystalline penicillin $\mathrm{G}$ at 6 -h intervals. The CSF Herpes simplex virus (HSV) 1-2, Epstein Barr virus (EBV), Cytomegalovirus (CMV), Human Herpesvirus 6 (HHV-6) and other multiplex PCR tests were negative. Weakness in the left arm and loss of vision in the left eye occurred on the $7^{\text {th }}$ day of monitoring. Muscle strength in the left arm was $3 / 5$. Control cranial computerized tomography (CT) and diffusion MRI revealed signal loss in the anterior horn of the lateral ventricle on ADC sequences [Fig.1], and restricted diffusion in the right parieto-occipital area from the apex to the temporal at the right basal ganglion level [Fig.2]. No findings of neuritis were determined at fundus examination. Low molecular weight heparin and

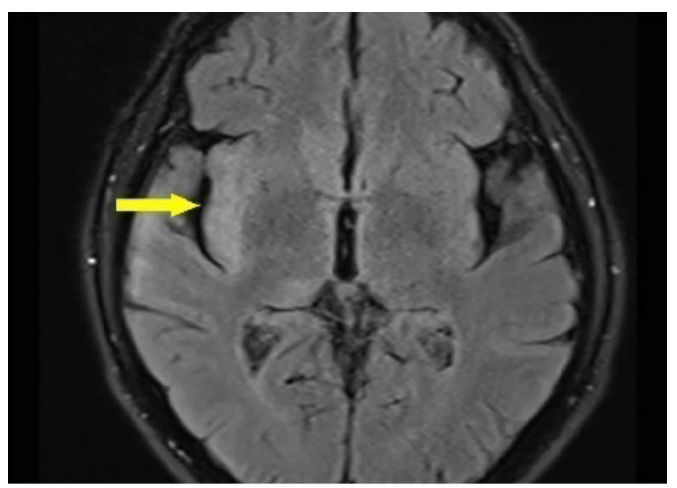

Fig.1: Axial FLAIR MRI was observed singal increases in right insular cortex.

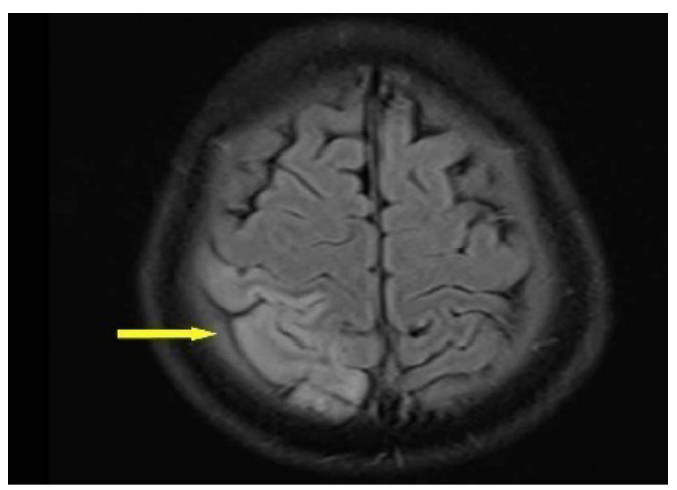

Fig.2: Axial FLAIR MRI was observed singal increases the right parietal lobe in the sections passing vertex. 
steroids were added to treatment. Penicillin G therapy was completed in 14 days. LP was repeated prior to discharge, and CSF VDRL was reported negative.

The patient was discharged in a clinically stable condition on the $4^{\text {th }}$ week of treatment. At follow-up after 3 months, clinical improvement was observed in the patient's psychotic and neurological symptoms, while MRI confirmed the infection. Volume loss in the right temporal lobe was observed on coronal FLAIR images, together with definition of the temporal horn of the right lateral ventricle [Fig.3], while the lesions in the right parietal lobe on axial FLAIR images and gliosis improved at control MRI after 3 months, and volume loss was observed in the gyri [Fig.4].

\section{Discussion}

Symptomatic late disease develops in approximately $30-40 \%$ of untreated syphilis cases [5]. Neurological characteristics may appear at the beginning of the disease process but are generally seen many years after primary infection. Neurosyphilis may remain asymptomatic in the early stages of the disease, and can produce symptoms in the form of meningitis, stroke, vertigo, optic neuritis and uveitis. In later periods neuropsychiatric findings associated with brain and spinal cord involvement or tabes dorsalis may be seen. Symptomatic findings of neurosyphilis are associated with meningovascular and parenchymatous involvement. Meningovascular syphilis is seen in $10 \%$ of all neurosyphilis cases, with peak development and pathological changes being observed at mean of 7 years following primary infection. Primary and secondary stages had not been identified in our case, and evaluation took place in the late neurosyphilitic period on the basis of neuropsychiatric symptoms [6,7]. Genuine nerve cell damage occurs in parenchymatous neurosyphilis. General paresis and tabes dorsalis are prominent findings. Psychiatric and neurological signs are observed in addition to

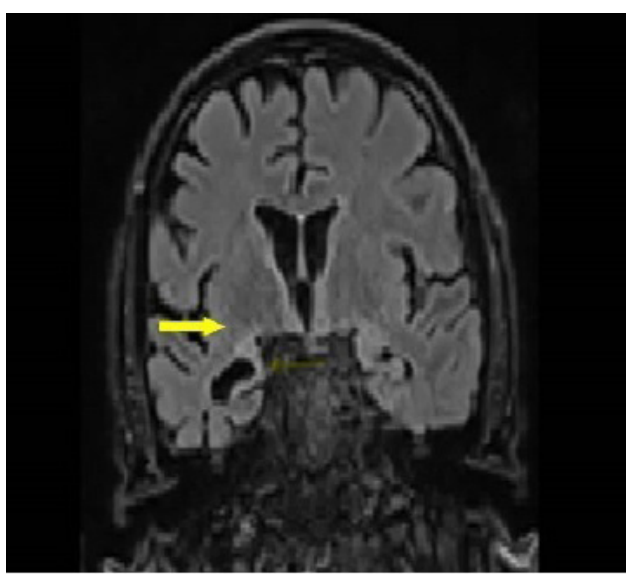

Fig.3: At the end of the third month showed volume loss and sclerosis in the right mesial temporal lobe, prominence in the lateral venticular temporal horn in FLAIR sequence MRI.

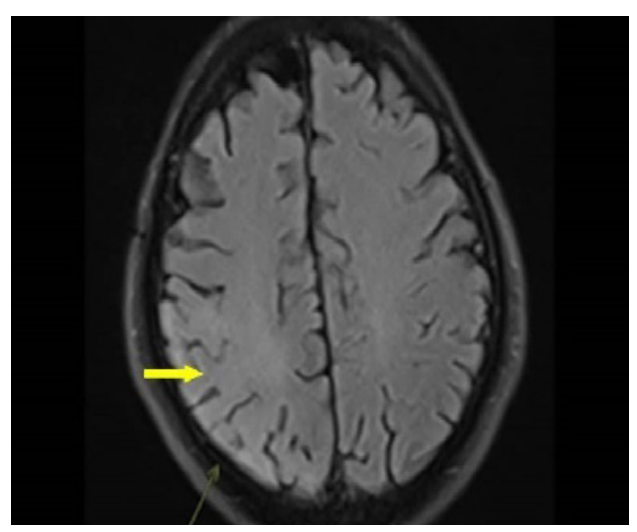

Fig.4: Healing of lesions in right parietal with gliosis and volume loss in gyrus in MRI.

diffuse parenchymal damage. Similar clinical findings were present in our patient (agitation and hallucination, followed by sleep sedation). Rare clinical malnifestations of neurosyphilis include complex partial seizure and hemiparesis [8-10].

Chewing motions in a case of neurosyphilis may be explained in terms of chewing-type complex partial status epilepticus (CPSE) and the mesial temporal location of syphilitic (luetic) vasculitis. At radiological tests investigating clinical findings, T2-weighted images will probably show cortical and subcortical hyperintensity, due to both cytotoxic and vasogenic edema. Images resemble those of HSV encephalitis. Short-term repetition 
of cerebral MRI and CSF examination may be useful at differential diagnosis of mesial temporal syndromes [8].

Diagnosis of neurosyphilis is based on risk history, personal history, clinical findings, and serological methods. In diagnostic terms, nontreponemal tests (VDRL and RPR) are used for screening purposes, while treponemal tests are used for confirmation (TPHA, MHA TP, and FTA ABS). TPHA or FTA positivity in CSF does not by itself confirm a diagnosis of syphilis, but negativity excludes such a diagnosis. Pressure is increased in CSF, glucose is low and protein is high. The leukocyte count is $10-500 \mathrm{~mm}^{3}$. An increase in mononuclear cells in CSF is significant. Our patient presented with a manifestation of acute encephalitis, and paralysis and complex partial seizure were observed. Neurosyphilis was based on differential diagnosis of encephalitis on the basis of CSF analysis and VDRL and TPHA positivity.

In terms of treatment, 10-14 day benzathine penicillin $\mathrm{G}$ at 18-24 MU/day is used in patients with neurological symptoms, irrespective of stage of neurosyphilis. Oral doxycycline $200 \mathrm{mg}$ /day for 14 days, tetracycline or erythromycin $500 \mathrm{mg} 4 \mathrm{x} 1$, or single-dose azithromycin $2 \mathrm{~g}$ /day for 14 days are used in patients with hypersensitivity. Response to treatment must be monitored with CSF, and a decreased cell count is particularly significant. Our patient received $4 \mathrm{MU}$ crystalline penicillin $\mathrm{G}$ i.v. at 6-h intervals. When post-treatment CSF findings were compared, we observed a decreased white cell count in lymphocyte predominance [6].

\section{Conclusion}

Neurogenic syphilis involvement should not be overlooked at differential diagnosis in patients presenting with clinical and radiological findings suggestive of herpes encephalitis. Untreated cases of primary and secondary syphilis will probably become tertiary and exhibit late findings.
Consideration of neurosyphilis in patients with neurological components such as chewing type complex partial seizure and stroke, and with psychotic symptoms such as agitation and hallucination, will be useful in terms of early diagnosis and in the approach to treatment.

Contributors: UK, AE, SO clinically managed the patient, wrote the manuscript. MEK, IEY edited the manuscript. MFI provided critical inputs into the case and did imaging. AE will act as study guarantor. All authors approved the final version of this manuscript.

Funding: None; Competing interests: None stated.

\section{References}

1. Drago F, Merlo G, Ciccarese G, Agnoletti AF, Cozzani E, Rebora A, et al. Changes in neurosyphilis presentation: a survey on 286 patients. J Eur Acad Dermatology Venereol. 2016;30:1886-1900.

2. Marra CM. Neurosyphilis. Continuum (Minneap Minn). Neuroinfectious Disease. 2015;21:1714-1728.

3. Saunderson RB, Chan RC. Mesiotemporal changes on magnetic resonance imaging in neurosyphilis. Intern Med J. 2012;42:1057-1063.

4. Nagappa M, Sinha S, Taly AB, Rao SL, Nagarathna S, Bindu PS, et al. Neurosyphilis: MRI features and their phenotypic correlation in a cohort of 35 patients from a tertiary care university hospital. Neuroradiology. 2013;55:379-388.

5. Kamath M, Rizvi M, O'Nions J, Brook G. A complex neurological presentation of syphilis. BMJ Case Rep. 2013 doi:10.1136/bcr-2013-200309.

6. Tramont EC. Treponema pallidum (Syphilis). In: Mandell GL, Bennett JE, Dolin R, eds. Principles and Practice of Infectious Diseases. $8^{\text {th }}$ edition. 3035-3053.

7. Ropper AH, Brown RH. Infections of the nervous system (bacterial, fungal, spirochetal, parasitic and sarcoidosis). Adams and Victor's Principles of Neurology. $8^{\text {th }}$ ed. New York: McGraw-Hill;2005:592-630.

8. Marano E, Briganti F, Tortora F, Elefante A, De Rosa A, Maiuri F, et al. Neurosyphilis with complex partial status epilepticus and mesiotemporal MRI abnormalities mimicking herpes simplex encephalitis. J Neurol Neurosurg Psychiatry. 2004;75:833.

9. Hooshmand H. Seizure disorders associated with neurosyphilis. Dis Nerv Syst. 1976;37:133-136.

10. Sinha S, Harish T, Taly AB, Murthy P, Nagarathna S, Chandramuki A. Symptomatic seizures in neurosyphilis: an experience from a university hospital in south India. Seizure. 2008;17:711-716. 